\title{
META-ANALYSIS OF CRASH RISK FACTORS AMONG OLDER DRIVERS: APPLICATION TO A MODEL PROGRAM OF DRIVER SCREENING
}

\author{
Karlene Ball, Virginia Wadley, Jerri Edwards, David Ball \\ University of Alabama at Birmingham \\ Department of Psychology and Center for Research on Applied Gerontology \\ Daniel Roenker \\ Western Kentucky University \\ Department of Psychology
}

In the absence of disease or impairment, there is no empirical evidence that subtle, agerelated changes in sensory or cognitive function affect older drivers' abilities to safely operate a motor vehicle. However, impairments that do affect driving occur with a higher prevalence in the older population.

This paper describes a meta-analysis of risk factors for automobile crash. Risks associated with compromises in visual acuity, contrast sensitivity, visual fields, useful field of view, and mental status are examined. Risks associated with medical conditions, medications, and physical limitations also are discussed.

Results demonstrate that visual acuity and contrast sensitivity are only weakly associated with crash risk, while cognitive variables and mental status measures are moderately associated with crash risk. A specific measure of The Useful Field of View, UFOV ${ }^{\circledR}$, is strongly associated with crash risk.

These results suggest that driver evaluations aimed at detecting unsafe drivers can be significantly improved. Specifically, evaluations can be improved by including a broader assessment of visual function, an assessment of cognitive function including the $\mathrm{UFOV}^{\circledR}$, an assessment of mental status, and an assessment of physical status. An evaluation incorporating these components might facilitate driver qualification or referral for appropriate intervention.

A driver screening evaluation program incorporating these components is currently underway. With over 2,000 older adult participants, results from this model program indicate that cognitive function, rather than visual or physical function, is most strongly associated with mobility outcomes. Thus, interventions to maintain or improve cognitive function may also help to sustain mobility. 